\title{
USO DE LINGUAGEM C++ EM COMPUTADOR EMBARCADO DE ALTA CAPACIDADE PARA EFICIENTIZAÇÃO ENERGÉTICA EM TRATORES
}

\author{
DEIVITY DO CARMO SANTOS ${ }^{1}$, EVANDRO CHARTUNI MANTOVANI', \\ ALÍPIO MONTEIRO BARBOSA² e WALTER LUIZ DE CASTRO MEWES ${ }^{3}$
}

\author{
${ }^{1}$ Laboratório de Mecanização Agrícola - Embrapa Milho e Sorgo Rod. MG 424 Km 45, Caixa Postal 285 ou 151, 35701-970, \\ Zona Rural, SeteLagoas-MG.dsantos1995@hotmail.com,evandro.mantovani@embrapa.br \\ ${ }^{2}$ Grupo de Modelagem, Automação e Controle - Centro Universitário de Sete Lagoas, \\ Av. Marechal Castelo Branco, 2765, 35701-242, Sete Lagoas-MG, Brasil.alipio.barbosa@unifemm.edu.br \\ ${ }^{3}$ Instituto de Ciências Agrárias/CAF - Universidade Federal de Viçosa. \\ RodoviaLMG818,km 6,35690-000,Florestal-MG,Brasil.mewes@ufv.b
}

Revista Brasileira de Milho e Sorgo, v.17, n.2, p. 340-352, 2018

\begin{abstract}
Resumo: A utilização de tratores e implementos agrícolas é amplamente difundida em todos os tipos de cultura, atendendo às necessidades das diferentes escalas de produção das propriedades rurais. Um dos principais desafios inerentes ao uso desse tipo de equipamento é a dificuldade de aperfeiçoar seu emprego, estabelecendo-se condições de operação, que propiciem o equilíbrio entre a alta capacidade de trabalho que a mecanização agrícola proporciona, o consumo adequado de energia e a sustentabilidade da área de produção. Este trabalho descreve as etapas de implementação de um sistema de aquisição de dados e visa avaliar o desempenho energético de um conjunto tratorimplemento, utilizando um computador de placa única baseado na arquitetura ARM e sensores diversos. Algoritmos foram escritos em linguagem de programação $\mathrm{C}++$ a fim de obter os sinais provenientes de cada um dos sensores e trata-los conforme sua natureza e grandeza física a ser mensurada. Após a etapa de programação, os sensores foram individualmente testados e calibrados em bancada e, posteriormente, o sistema como um todo foi testado no conjunto trator-implemento. O sistema mostrou-se uma ferramenta válida para auxiliar na tomada de decisão em operações de campo envolvendo máquinas agrícolas.
\end{abstract}

Palavras-chave: mecanização agrícola, instrumentação eletrônica, sistemas embarcados.

\section{HIGH CAPACITY EMBEDDED COMPUTER WITH C++ FOR EVALUATING TRACTOR PERFORMANCE AT FIELD}

\begin{abstract}
Agricultural machinery and implements have been widely used in all crops, supporting the needs of several productive scales. One of the main challenges to the use of such equipment is the difficulty of optimizing its usage, establishing operational conditions that allow the balance between the high performance provided by agricultural mechanization and the sustainability of the planting area. This paper describes the steps in implementing a high capacity acquisition system that allows evaluating the energetic performance of a set of tractor-implement using a single-board computer based on ARM architecture and sensors. The $\mathrm{C}++$ programming language was chosen to implement the algorithms that read the signals provided by sensors and treat them according to the measured physical quantity. Thereafter, the sensors were individually tested in the laboratory and also at field with all sensors connected to the embedded computer. The system proved to be a valid tool to aid in decision making in operations with agricultural machinery. Keywords: agricultural mechanization, electronic instrumentation, embedded systems.
\end{abstract}


A produção agrícola cresceu consideravelmente nas últimas décadas com as tecnologias agronômicas disponibilizadas pela pesquisa e com suporte da mecanização agrícola. Os agricultores puderam realizar o preparo do solo, a semeadura, cultivo e colheita dentro do cronograma de cada cultura. Apesar dos avanços e dos ganhos produtivos, a mecanização agrícola trouxe consigo uma série de impactos sobre a área de plantio, como por exemplo, a compactação do solo provocada pelo peso do maquinário e excesso de patinagem das rodas do trator (Houmy et al., 2013). Torna-se então necessária a adoção de estratégias que permitam usufruir da mecanização agrícola de forma adequada e sustentável, inclusive no consumo de energia. É possível chegar ao equilíbrio entre mecanização agrícola e manejo adequado do solo, contudo, para tal é imprescindível conhecer o sistema de manejo e definir os parâmetros de operação da máquina, visando a alta capacidade efetiva de trabalho sem deteriorar o solo (Mantovani, 1986).

Para avaliar o desempenho dos equipamentos agrícolas, torna-se necessária a realização de medições das variáveis de interesse durante a operação do maquinário, que posteriormente serão usadas para análise de desempenho na tomada de decisão (Mantovani et al., 1999). Com esse intuito, sistemas automáticos de aquisição vêm sendo desenvolvidos utilizando as mais diversas plataformas, como mostrado por Garcia et al. (2005).

A crescente evolução tecnológica dos microcontroladores e computadores embarcados tem propiciado o surgimento de diversas soluções voltadas ao agronegócio e demais setores da economia (Lamborelle \& Álvarez, 2017). Tais dispositivos aparecem como uma proeminente opção para criar soluções personalizadas para atender às demandas específicas de cada usuário, uma vez que permitem interagir com uma vasta gama de sensores. Além disso, a conectividade provida pelas plataformas de desenvolvimento microprocessadas possibilitam de transferência de dados para estações remotas, em tempo real, facilitando o trabalho de campo.

Uma série de sistemas embarcados tem sido empregada nas mais diversas etapas produtivas, como apoio à prática da agricultura de precisão. Entre as possíveis aplicações, Rubhini et al. (2014) destacam o controle de pragas e plantas daninhas, além dos estudos de variabilidade das características do solo e plantas. A geoespacialização da área de produção, possível devido ao aumento da precisão dos aparelhos de GNSS (Global Navigation Satellite Systems), possibilita que sensores mensurem pontualmente as grandezas de interesse, em tempo real, durante as operações de campo (Grego et al., 2014). Desta forma, pode-se ter a variabilidade espacial da energia consumida no campo através do mapeamento georeferenciado durante a execução dos trabalhos e avaliar se os valores estão de acordo com os valores técnicos recomendados.

Os dispositivos microprocessados possuem uma considerável capacidade de processamento e, em alguns casos, de armazenamento. Todavia, estes possuem limitações significativas se comparados aos computadores convencionais (Molloy, 2014). Por esta razão, a programação de sistemas embarcados deve ser a mais compactada possível. Uma das possíveis soluções para aperfeiçoar os algoritmos é a utilização de linguagem "Assembly", uma vez que a programação é feita exatamente com os registradores e instruções do processador definidas pelo fabricante, sendo assim, classificada como uma linguagem de baixo para médio nível de abstração em relação à linguagem de máquina. 
Entretanto, a programação em "Assembly" exige profundo conhecimento das especificidades do hardware, além de seus códigos geralmente serem maiores e mais complexos em comparação com as linguagens de programação que possuem um alto nível de abstração, mais parecidas com a linguagem humana e menos parecida com a linguagem de máquina.

As linguagens $\mathrm{C}$ e $\mathrm{C}++$ são boas alternativas quando não se deseja escrever os códigos de programação diretamente em linguagem Assembly, uma vez que estas contêm recursos das linguagens baixo nível, como o acesso aos registradores do processador e a manipulação de bits. Em contrapartida, pode-se programar com a facilidade razoável que as linguagens de alto nível apresentam. Por serem multipropósito, grande vantagem de programar utilizando essas linguagens é a compatibilidade com a maior parte das arquiteturas de sistemas, além de possuir quantidade considerável de bibliotecas existentes e acessíveis.

A microeletrônica aplicada a sistemas agrícolas, a conectividade dos sistemas, a automação, a agricultura de precisão e o uso de big data são algumas das características de uma nova fase na agricultura. Com auxílio desses recursos e demais avanços tecnológicos recentes, como o aumento da precisão dos aparelhos receptores de GNSS para menos de 2,5 cm, torna-se cada vez mais difundido o emprego de veículos autônomos, robôs, bem como outros aparatos tecnológicos nas operações de campo. Essa abordagem é tratada na agricultura como "Smart farming".

O objetivo deste trabalho foi desenvolver um sistema de aquisição de dados, de baixo custo, embarcado em um conjunto trator-implemento, visando a redução do consumo de energia durante operações de conjuntos trator-implemento.

\section{Material e Métodos}

Este trabalho foi desenvolvido no Laboratório de Mecanização, da Embrapa Milho e Sorgo, cujas coordenadas geográficas são $19^{\circ} 26^{\prime} 41,5^{\prime \prime} \mathrm{S}$ e $44^{\circ}$ 09' 51,7' W, com altitude de 732 metros. Os testes de campo foram realizados na área experimental, em um Latossolo Vermelho Escuro, sendo o teor de umidade do solo monitorado, em todos os testes, com amostras compostas, do perfil cortado.

O sistema proposto para este trabalho foi desenvolvido para um conjunto trator-implemento, com diferentes sensores conectados a um computador de bordo, que permitiu medir as variáveis mais importantes durante a operação no campo, visando à otimização do consumo de energia.

Para a realização dos testes, o sistema foi instalado em um trator Massey Ferguson 292 Turbo 4x4, de $77 \mathrm{~kW}$ de potência, em conjunto com uma grade aradora Tatu Controle Remoto, de 14 discos de 26 polegadas, pesando $1440 \mathrm{~kg}$.

\section{Sistema de aquisição de dados}

O sistema de aquisição de dados foi composto pelos seguintes equipamentos:

1. Um computador embarcado, de placa única, Beaglebone Black Rev. C;

2. Uma célula de carga Kratos CCI, 196 kN;

3. Um sensor Ultrassônico HC-SR04;

4. Um módulo GPS Ublox NEO-6M;

5. Dois sensores de proximidade indutivos Schoenbuch IBCT 1812;

6. Dois medidores de combustível Micro Ovall

\section{LS 4150;}

7. Radar Dickey John II. 


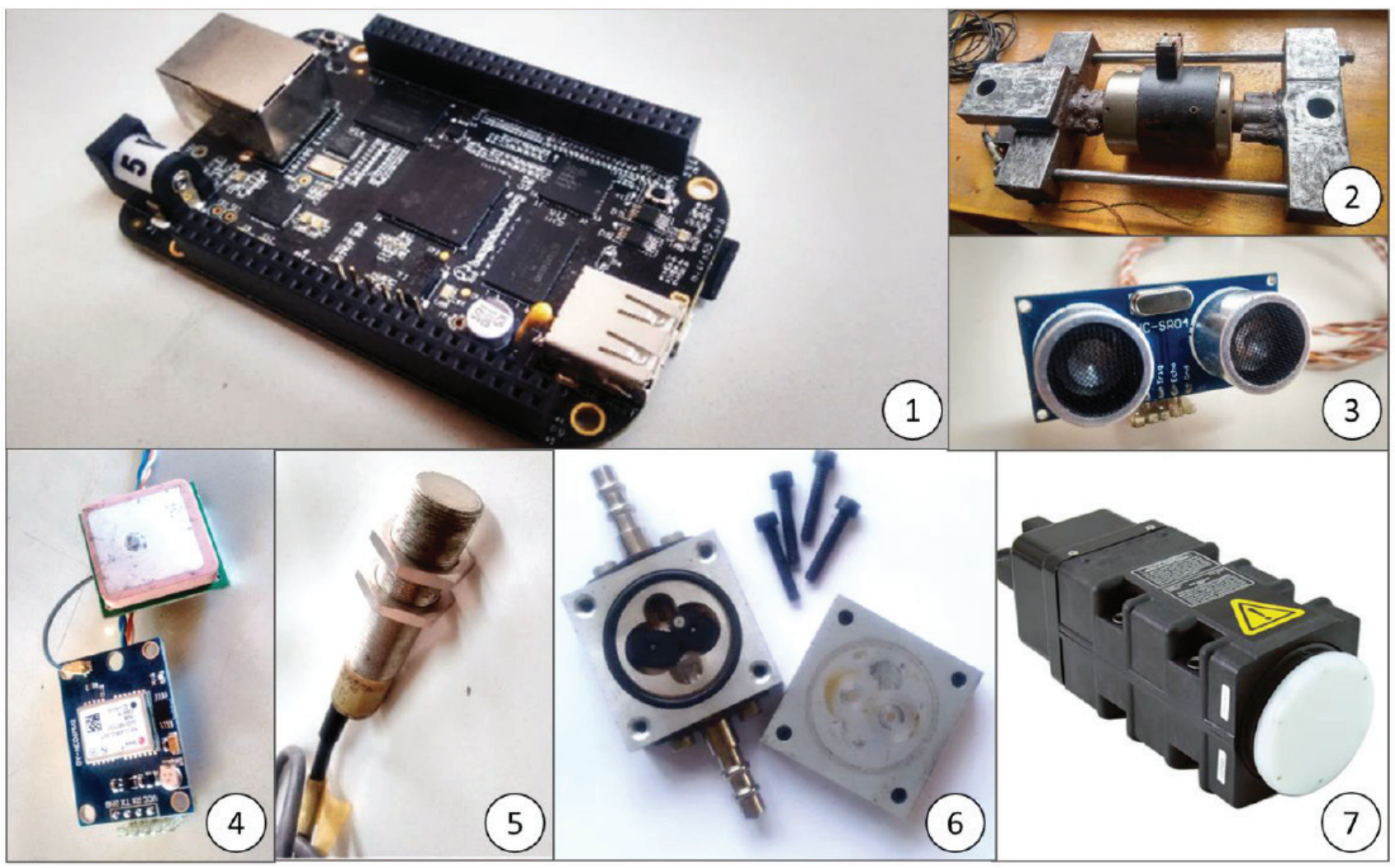

Figura 1. Visão geral do sistema. 1) Beaglebone Black, 2) Célula de Carga, 3) Sensor Ultrassônico, 4) Módulo GPS, 5) Sensores indutivos, 6) Medidores de vazão e 7) Radar Dickey John II.

O computador embarcado de placa única Beaglebone Black Rev. C foi utilizado com o sistema operacional Linux Debian 7.8 e sua finalidade era receber, processar e armazenar os sinais provenientes dos sensores por meio de suas entradas digitais, analógicas e canais de comunicação UART (Universal asynchronous receiver/transmitter). É possível utilizar até 65 canais como entradas ou saídas digitais, sendo que os níveis lógicos baixo e alto são, respectivamente, 0 e 3,3 $\mathrm{V}_{\mathrm{DC}}$. O computador possui 7 entradas analógicas independentes e admitem valores de tensão entre 0 e 1,8 $\mathrm{V}_{\mathrm{DC}}$, as mesmas estão ligadas diretamente a conversores analógico-digital (ADC) de 12 bits, que convertem a tensão de entrada em valores discretos de 0 a 4095 .
Os sensores componentes do sistema de aquisição de dados convertiam as grandezas físicas de interesse em sinais elétricos (digitais e analógicos), a fim de que pudessem ser processados pelo computador embarcado. Uma célula de carga Kratos CCI, com capacidade para $196 \mathrm{kN}$, foi instalada entre o trator e o implemento para medir a força de tração demandada pelo implemento. Este é um dispositivo constituído por elementos resistivos sensíveis à deformação mecânica (strain gages), que internamente estão configurados em um arranjo conhecido como Ponte de Wheatstone. O transdutor produz um sinal contínuo e analógico, proporcional à força resultante na barra de tração. 
O sensor ultrassônico HC-SR04 mensura a profundidade de trabalho através da emissão de ondas ultrassônicas, 8 ciclos a $40 \mathrm{kHz}$. Tal dispositivo possui quatro pinos: $\mathrm{V}_{\mathrm{DC}}$, GND, "echo" e "trigger". O pino de "trigger" é um de gatilho que promove o disparo do sinal ultrassônico. Ao incidir em algum obstáculo, as ondas ultrassônicas emitidas são ecoadas de volta ao sensor. Uma vez que o eco é recebido, os circuitos internos do sensor colocam o pino "echo" em nível lógico alto durante tempo proporcional ao intervalo decorrido entre a emissão e recepção do sinal ultrassônico. Segundo as informações do datasheet do HC-SR04, a distância entre o sensor e o obstáculo é dada, em milímetros, pela Equação 1 .

$$
p=\frac{t}{5,844}
$$

em que $p$ é a profundidade medida em milímetros e $t$ é o tempo que o pino "echo" permaneceu em nível lógico alto, em microssegundos.

O módulo GPS Ublox NEO-6M obteve informações bastante pertinentes, como a posição geográfica e a velocidade linear do trator. As informações em questão eram transmitidas do módulo para o computador embarcado através de comunicação serial (UART).

Os sensores de proximidade indutivos Schoenbuch IBCT 1812 foram instalados nas rodas traseiras do trator, para medir a patinagem das rodas motoras quando em trabalho com implementos. Esses dispositivos são capazes de detectar a presença de massas metálicas por meio da emissão de um campo magnético produzido por um oscilador e uma bobina. Ao ser penetrado por uma massa metálica, o campo magnético perde energia suficiente para que a variação do campo seja detectada pelos circuitos internos que, por sua vez, promovem a inversão do nível lógico na saída de sinal do sensor.
Liljedahl et al. (1989) apresentaram uma das formas de se calcular a patinagem $(S)$ da máquina agrícola, através da relação entre as velocidades teórica $\left(v_{t}\right)$ e real $\left(v_{a}\right)$ do trator. Utiliza-se a Equação 2.

$$
S=1-\frac{v_{a}}{v_{t}}
$$

Para obter a velocidade teórica por meio dos sensores indutivos, imãs foram dispostos ao longo das rodas motoras a uma distância adequada do sensor. Desta forma, um pulso é emitido cada vez que um dos imãs passa pelo indutivo. Logo, velocidade teórica é dada pela Equação 3.

$$
v_{a}=\frac{\omega D}{2}=\frac{n_{p u l s o s} D \pi}{n_{\text {imås }} t}
$$

em que w e $D$ são, respectivamente, o deslocamento e o diâmetro da roda, $n_{\text {pulsos }} n_{\text {pulsos é a quan- }}$ tidade de pulsos emitidos durante o percurso, $n_{\text {imãs }}$

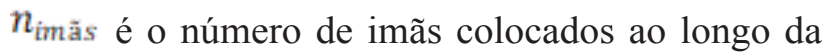
roda e $t$ é o tempo decorrido durante a contagem de pulsos.

O medidor de combustível, Micro Ovall II LS 4150, possui uma câmara em seu interior, onde estão situadas duas engrenagens ovais que giram em função do fluxo que passa por elas, de forma a completar uma volta a cada 1 mililitro de combustível deslocado. Uma dessas engrenagens possui um pequeno imã, responsável por fechar, por meio de seu campo magnético, o contato elétrico presente na estrutura do dispositivo. Para tanto, foi instalado um sensor na entrada da bomba de combustível e outro no retorno para o tanque, para medir o consumo através da diferença de leitura entre os sensores.

A vazão instantânea $(Q)$ foi obtida em mililitro por unidade de tempo através da Equação 4. Por fim, 
$t$ é o tempo decorrido até que as engrenagens completem uma volta.

$$
Q=\frac{1}{t}
$$

O Radar Dickey-John II, utilizado para medir a velocidade de deslocamento do conjunto trator-implemento, apresenta o seu princípio de funcionamento na emissão de microondas eletromagnéticas em direção ao solo e recepção do eco produzido. $\mathrm{O}$ deslocamento foi mensurado com base no tempo entre a emissão e recepção das microondas, a exemplo do sensor ultrassônico descrito anteriormente. Os circuitos internos produzem um pulso elétrico na saída para cada centímetro deslocado pelo veículo no qual o dispositivo está instalado. A velocidade (em distância por unidade de tempo) foi obtida a partir do sinal emitido pelo radar por meio da Equação 5.

$$
u=\frac{0,01 m}{t}
$$

em que $u$ é a velocidade a ser obtida, e $t$ o tempo decorrido entre dois pulsos elétricos provenientes do radar. O deslocamento (em metros), por sua vez, também pode se ser obtido, dividindo-se o número de pulsos por 10 .

Algumas adequações foram necessárias nessa fase para atender às especificações de hardware do computador embarcado e melhorar a qualidade da leitura de sinal. O radar e os sensores indutivos emitem sinais de saída com tensão igual a $12 \mathrm{~V}_{\mathrm{DC}}$, enquanto a Beaglebone trabalha com o nível lógico de $3,3 \mathrm{~V}_{\mathrm{DC}}$ em suas entradas digitais. Para adequar o sinal, foi utilizado um circuito eletrônico para fazer a conversão do sinal.

Devido à baixa tensão que é fornecida nos terminais de saída da célula de carga $(2 \mathrm{mV} / \mathrm{V})$, foi necessário utilizar um amplificador operacional instrumental. O amplificador, cujo esquema eletrônico é mostrado na Figura 2 foi construído a partir de dois circuitos integrados, OP77EZ, e amplifica em 23 vezes o sinal produzido pela célula de carga.

\section{Desenvolvimento do programa gerenciador de leituras dos sensores:}

O programa foi desenvolvido com intuito de gerenciar a leitura de sinais provenientes de todos os sensores. Os códigos-fonte da programação foram

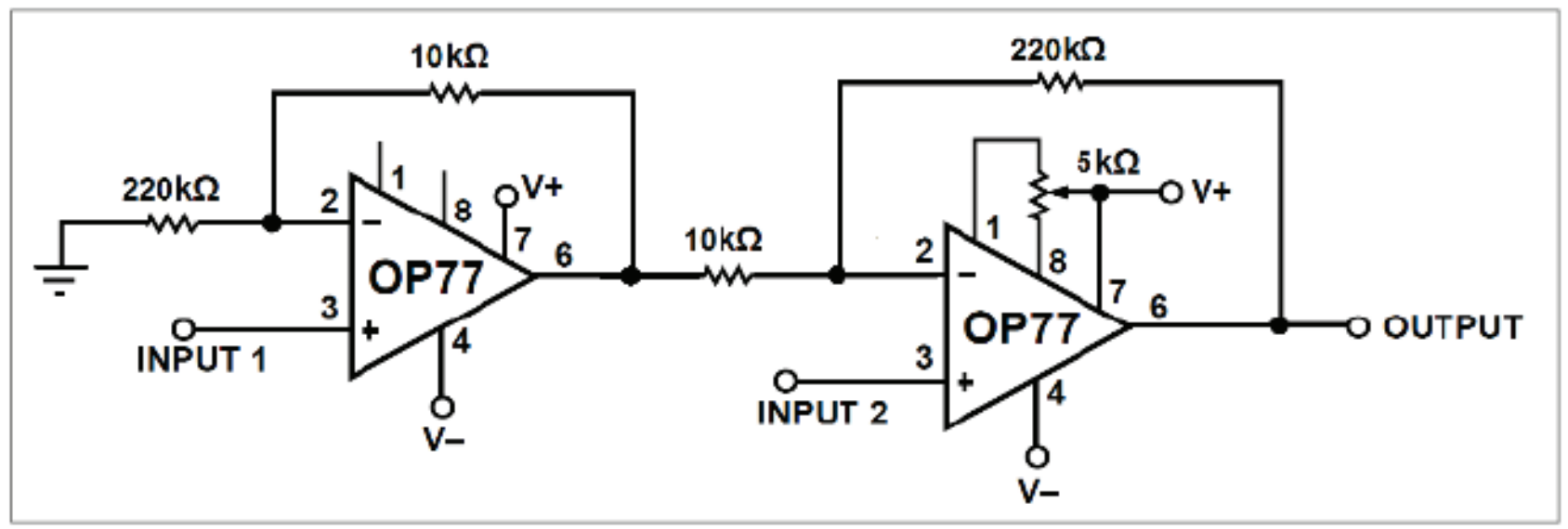

Figura 2. Esquema eletrônico do amplificador instrumental com ganho igual a 23. O potenciômetro conectado aos pinos 1, 7 e 8 é destinado ao ajuste da tensão de offset. 
implementados utilizando linguagens $\mathrm{C}$ e $\mathrm{C}++$. Depois de compilados, as aplicações geradas permitiram realizar a leitura do sensor para qual foi programada, bem como armazenar tais leituras em arquivos de texto, dentro do tempo pré-programado.

Os sensores indutivos, radar e medidores de consumo, emitem sinais de saída digitais na forma de uma onda quadrada ou trem de pulso. Por essa razão, um mesmo código foi utilizado para realizar a leitura desses sensores, que consiste em obter o tempo decorrido entre duas bordas de subida, um período da forma de onda. A partir do tempo aferido foi possível tratar os registros a fim de obter a grandeza mensurada pelo sensor, utilizando suas respectivas equações, conforme mostrado anteriormente.

Para leitura do sinal analógico oriundo da célula de carga um algoritmo foi implementado para registrar o valor instantâneo de tensão presente no $\mathrm{ADC}$ ao qual o transdutor de força está ligado. Tais valores foram obtidos em intervalos de 1,25 milissegundos, durante 1 segundo. Ao final deste período, a média das leituras instantâneas (valores discretos de 0 a 4095) foi convertida novamente em um valor de tensão, através da Equação 6 e, posteriormente, a força de tração pôde ser obtida por meio da curva de calibração da célula de carga, que será apresentada posteriormente.

$$
V=\frac{1,8 \bar{x}_{a d c}}{4095}
$$

em que $\mathrm{V}$ é o sinal de tensão a ser obtido, em volts, e $\bar{x}_{a d c}$ é a média das leituras instantâneas do ADC, realizadas durante 1 segundo.

Seguindo as especificações do sensor ultrassônico HC-SR04, um algoritmo foi implementado para medir a distância entre o sensor e o solo e, consequentemente, a profundidade de trabalho. A execução do arquivo gerado após compilação do código fazia com que o pino de trigger fosse mantido em nível lógico alto durante $10 \mu \mathrm{s}$, a fim de disparar o sinal ultrassônico. Em seguida, foi feita a leitura do sinal do pino echo e contabilizado quanto tempo este permaneceu em nível lógico alto. Por fim, tal tempo é convertido em distância utilizando a Equação 1. Uma particularidade da programação para esse sensor foi o uso das unidades de tempo real do computador embarcado para garantir maior precisão das medições. As unidades programáveis de tempo real, ou PRU (programmable real-time unit), são portas programáveis como entrada ou saída digital de alta velocidade e baixíssima latência.

Finalmente, para o módulo GNSS foi necessário implementar um código que realizasse a leitura dos dados disponibilizados via comunicação UART. As informações geradas, de horário UTC, latitude, longitude e velocidade, foram enviadas para o computador embarcado, usando o padrão das sentenças NMEA (National Marine Electronics Association), atualizadas a cada 1 segundo. Após compilar o código de programação, o executável gerado promoveu a leitura do dispositivo utilizando um dos canais UART do computador embarcado.

Para controlar o início e o final da execução das aplicações mencionadas anteriormente, uma interface gráfica foi desenvolvida com os softwares Visual Studio Community 2015 e o Qt Creator 4.2.1, com as seguintes telas: Leitura dos sensores, Configurações de data e hora e Terminal Linux. Na Figura 2 é apresentado o fluxograma simplificado do funcionamento do sistema de aquisição de dados. 


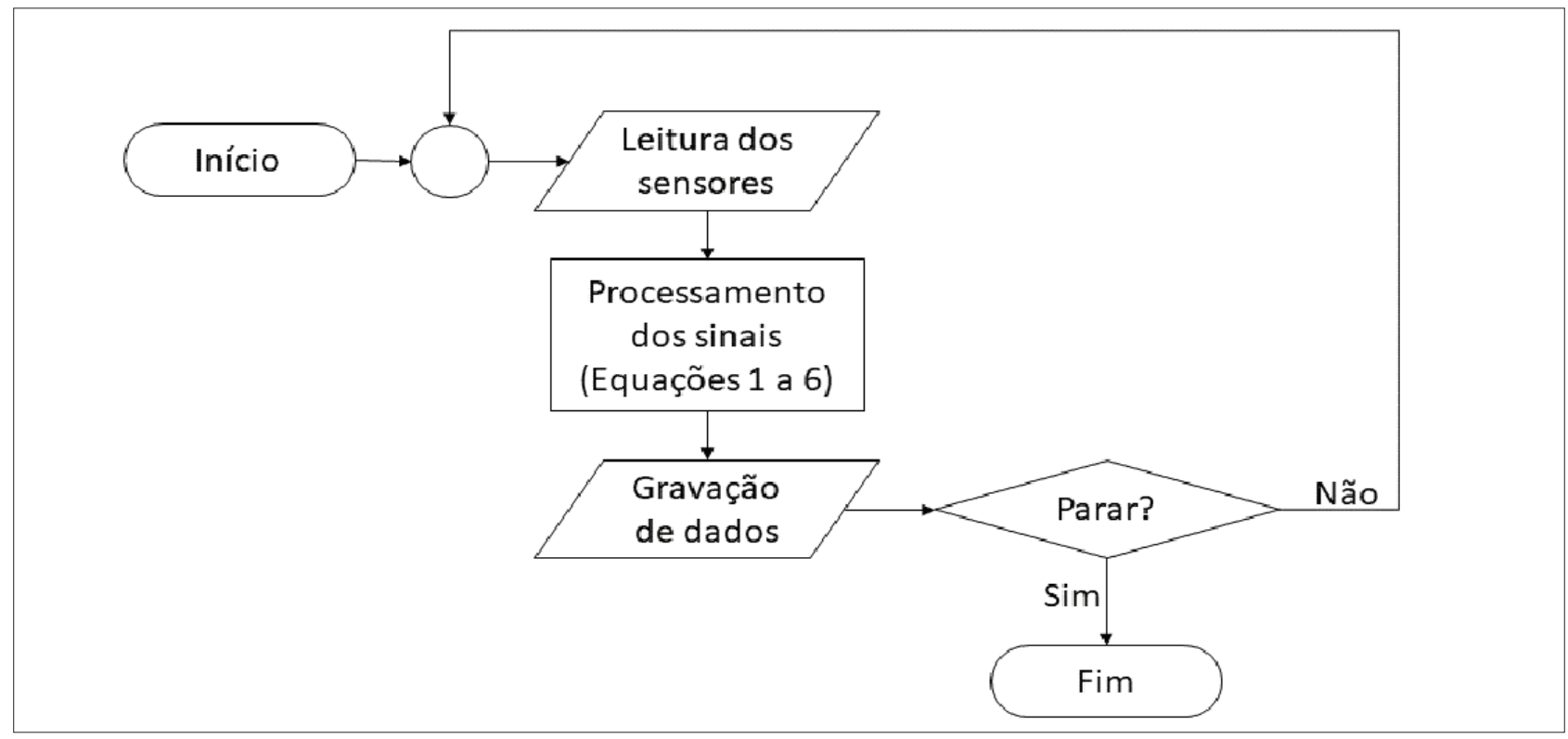

Figura 3 - Fluxograma simplificado do funcionamento do sistema.

\section{Resultados e Discussão}

A programação utilizada no sistema foi implementada de modo a atender aos diferentes tipos de sensores e respectivos tempos de programação. A eficiência do sistema de aquisição de dados foi testada inicialmente no laboratório e, posteriormente, no campo, para saber se os sensores estavam emitindo sinais coerentes com os resultados técnicos esperados. Desta maneira, serão apresentados a seguir os resultados dos dados coletados em campo pelos sensores, com as respectivas analises para validação do sistema automático de aquisição de dados:

\section{Programação do Sistema de Aquisição de Dados}

Diferente dos microcontroladores, os computadores embarcados, a exemplo do Beaglebone Black, permitem a execução simultânea de várias aplicações, devido à presença de um sistema operacional para gerenciar entradas e saídas, bem como a memória, processadores e demais periféricos do dispositivo. Diante disso, tornou-se possível que cada sensor fosse programado individualmente, para que seus respectivos sinais pudessem ser lidos e armazenados de forma independente.

Apesar de serem executadas separadamente, as aplicações devem estar sincronizadas, uma vez que as grandezas mensuradas através dos sensores são complementares. Para tal, em todos os códigos foi adicionada uma instrução em comum, que tem por finalidade coordenar as leituras, utilizando-se o horário local do sistema operacional do microcomputador para definir o intervalo e o momento da aquisição de dados.

O desenvolvimento da interface gráfica, mostrada na Figura 3, permitiu iniciar e parar a execução da aquisição de dados de todos os sensores, facilitando para o usuário, devido à complexidade das operações internas do sistema. Além disso, o controle do computador embarcado pode ser realizado através do 
Terminal Linux e de botões que permitem desligar e reiniciar o mesmo. Isso permitiu o acesso e execução de comandos nativos ao sistema operacional Linux, sem a necessidade de utilizar outros programas para este fim.

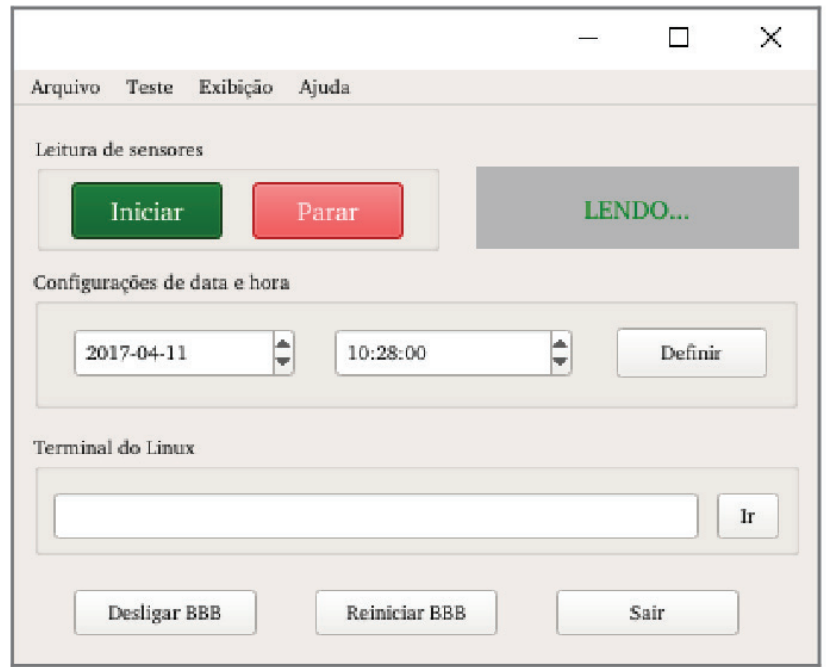

Figura 4. Painel com os componentes da interface gráfica.

Conforme mostrado na interface gráfica, as informações de data e hora da aquisição permitiram a organização dos arquivos de dados obtidos durante as atividades executadas pelo conjunto trator-implemento, no campo. Desta forma, o sistema de aquisição de dados facilitou o trabalho nos testes de campo, uma vez que as informações coletadas seguiam uma ordem previamente escalonada e a única coisa que precisou ser feita foi marcar o horário de início de cada tratamento, mostrado na tela da interface acima, 11/04/2017 e início às 10h28min.

\section{Patinagem}

Para medir a patinagem, foram instalados sensores indutivos nas rodas motoras do trator. Ao longo da roda esquerda foram alocados 3 imãs, enquanto na roda direita foram 6 imãs. A razão da utilização de um número maior de imãs na roda direita foi para comparar e avaliar a qualidade da informação gerada pelo sensor. Os dados obtidos indicaram que quanto maior a quantidade de pontos de contato, maior a precisão da medida, uma vez que a distância percorrida necessária para a emissão de um pulso será menor, reduzindo assim a possibilidade de que um ciclo não se complete durante a leitura. Com o trator em piso firme e sem carga, os sinais procedentes dos sensores indutivos foram registrados enquanto 100 metros eram percorridos, a exemplo do teste realizado para avaliar o deslocamento através do módulo GPS e do radar. Para calcular o deslocamento utilizou-se a Equação 3, sendo o diâmetro das rodas às quais os sensores foram instalados de aproximadamente 1,43 m. A velocidade média também pôde ser obtida, com base no tempo de duração do trajeto. A Tabela 1 mostra os resultados do teste realizado em 3 repetições:

Tabela 1. Avaliação do deslocamento por meio do sensor de proximidade indutivo instalado nas rodas direita e esquerda. Testes realizados em três repetições.

\begin{tabular}{|c|c|c|c|c|c|}
\hline Roda & Repetição & Pulsos & Duração (s) & Deslocamento (m) & Velocidade média $(\mathrm{m} / \mathrm{s})$ \\
\hline \multirow{3}{*}{ ๘ } & 1 & 134 & 42 & 100,33 & 2,39 \\
\hline & 2 & 132 & 56 & 98,83 & 1,76 \\
\hline & 3 & 134 & 55 & 100,33 & 1,82 \\
\hline \multirow{3}{*}{ 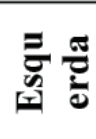 } & 1 & 66 & 42 & 98,83 & 2,35 \\
\hline & 2 & 66 & 56 & 98,83 & 1,76 \\
\hline & 3 & 67 & 55 & 100,33 & 1,82 \\
\hline
\end{tabular}


Durante a avaliação dos sensores indutivos, a velocidade média também foi simultaneamente obtida a partir módulo GNSS, para fins de comparação. Os valores obtidos na primeira, segunda e terceira repetição foram, respectivamente, $2,34 \mathrm{~m} / \mathrm{s}, 1,76 \mathrm{~m} / \mathrm{s}$ $1,81 \mathrm{~m} / \mathrm{s}$. Uma vez que os testes foram feitos em um piso firme, as velocidades teórica e real deveriam ser muito próximas, pois nessas condições a patinagem é praticamente nula. As médias entre as velocidades obtidas a partir dos sensores indutivos nas rodas esquerda e direita, de uma mesma repetição, foram utilizadas como velocidade teórica, enquanto as velocidades reais foram informadas pelo GNSS. A partir da Equação 3 e dos valores mostrados acima, os valores de patinagem calculados para as repetições 1,2 e 3 foram, respectivamente, $1,2 \%, 0 \%$ e $0,5 \%$.

Dada a proximidade dos valores obtidos utilizando-se diferentes dispositivos, os resultados demonstraram que os sensores de proximidade indutivos são sensíveis o bastante para calcular a velocidade teórica das rodas motoras. Desta forma, a patinagem pôde ser obtida a partir da equação 2 , utilizando também a velocidade informada pelo GNSS como a velocidade real do conjunto trator-implemento.

Portanto, para se obter os valores ainda mais próximos da patinagem real, pode-se aumentar a resolução da medição dos sensores de proximidade indutivos, alocando-se mais imãs ao longo da roda. Quanto maior a quantidade de pontos de sensibilização do sensor indutivo, menor o erro percentual.

\section{Força na barra de tração}

A calibração da célula de carga foi realizada de forma estática. O dispositivo foi alimentado com $1 \mathrm{~V}_{\mathrm{DC}}$ e submetido a diferentes valores de força, compreendidos entre 0 a $160 \mathrm{kN}$. Com os resultados obti- dos, utilizou-se o método da regressão linear para levantar um modelo matemático para a célula de carga. Chegou-se à Equação 7.

$$
y=0,0113125 x
$$

Em que $y$ é a tensão de saída em milivolts ( $\mathrm{mV}$ ) e $x$ a força de tração em quilonewton $(\mathrm{kN})$. O desvio padrão encontrado entre o modelo e as amostras aferidas durante a calibração foi de $0,01 \mathrm{mV}$. Sendo assim, a potência mecânica instantânea na barra de tração foi obtida multiplicando-se a força e a velocidade de um mesmo intervalo de tempo.

\section{Consumo de combustível}

O consumo de combustível foi obtido pela diferença entre as medições instantâneas dos dois sensores de combustível instalados no trator. Os testes realizados consistiram em registrar o consumo ao longo do tempo, sob condições similares e diferentes rotações do motor: 1700, 1900 e $2100 \mathrm{rpm}$. O trator Massey Ferguson 292 Turbo 4x4 em conjunto com a grade Aradora Tatu Controle Remoto, em um Latossolo Vermelho Escuro. Os resultados obtidos nos testes de campos estão mostrados na Tabela 2.

Conforme especificações técnicas do trator utilizado, os valores obtidos encontram-se dentro da faixa aceitável de consumo para o mesmo. As diferenças de consumo para uma mesma rotação deveram-se principalmente à não uniformidade do solo e demais condições de trabalho. O teor de água no solo, por exemplo, pode impactar diretamente na potência mecânica demandada pelo implemento, por maior ou menor adesão do contato do pneu com o solo. Apesar disso, foi possível perceber o comportamento similar do consumo de combustível ao longo das repetições, indicando que o sensor pode ser utilizado adequada- 
Tabela 2. Consumo médio de combustível, em 1/h, medido para três rotações e três repetições.

\begin{tabular}{c|c|c|c}
\hline Repetição & $\mathbf{1}$ & $\mathbf{2}$ & $\mathbf{3}$ \\
\hline Medições & & 8,08 & 8,38 \\
\hline Consumo (l/h) - 1700 rpm & 7,74 & 10,08 & 10,74 \\
\hline Consumo (1/h) - 1900 rpm & 10,20 & 11,10 & 11,56 \\
\hline Consumo (l/h) - 2100 rpm & 10,76 & 14,50 & 17,07 \\
\hline Teor de água do solo (\%) & 14,11 & 9,0 & 10,1 \\
\hline Profundidade Média (cm) & 8,4 & & \\
\hline
\end{tabular}

mente para indicar o consumo de combustível durante os trabalhos do conjunto no campo.

\section{Potência do motor:}

A potência foi obtida indiretamente na TDP devido à dificuldade de se obter instrumentação para medição nas rodas, o que seria mais adequado. A potência fornecida pelo motor foi determinada indiretamente através de metodologia realizada por Mantovani et al. (1999). A Figura 4 mostra as curvas de calibração obtidas com dinamômetro em um trator Massey Ferguson 292 Turbo 4x4, com consumo de combustível em $1 / \mathrm{h}$ e potência na tomada de potência (TDP) em kW. Desta forma, o valor de potência indireto foi obtido nas diferentes rotações do motor através dos diferentes valores de consumo de combustível, em litros por hora, mostrados na Tabela 3.

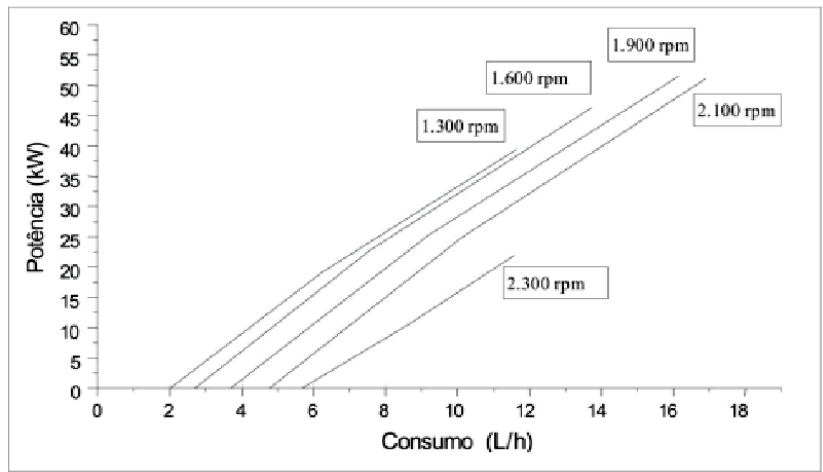

Figura 4 - Curvas de Consumo de combustível (1/h) x Potência (kW) do trator Massey Ferguson 292 Turbo 4x4 (Mantovani et al., 1999).
As Equações 8, 9 e 10, obtidas a partir das curvas de calibração mostradas na Figura 4, foram utilizadas para determinar a potência fornecida pelo motor em 1700, 1900 e $2100 \mathrm{rpm}$, respectivamente.

$$
\begin{aligned}
& P_{1700}=3,81 c-7,23 \\
& P_{1900}=3,78 c-9,4 \\
& P_{2100}=3,87 c-14,18
\end{aligned}
$$

em que a potência $\left(\mathrm{P}_{\mathrm{rpm}}\right)$, em quilowatts, é calculada em função do consumo (c), em litros por hora. Finalmente, na Tabela (3) são apresentados os valores de potência fornecida pelo motor, calculados para os mesmos valores de consumo mostrados na Tabela 2.

Tabela 3. Potência média fornecida pelo motor, em $\mathrm{kW}$, calculado para três rotações diferentes do motor. Testes realizados em três repetições.

\begin{tabular}{c|c|c|c|}
\hline \multirow{2}{*}{ Repetição } & \multicolumn{3}{|c|}{ Potência fornecida (kW) } \\
\cline { 2 - 4 } & $\mathbf{1 7 0 0} \mathbf{~ r p m}$ & $\mathbf{1 9 0 0} \mathbf{~ r p m}$ & $\mathbf{2 1 0 0} \mathbf{~ r p m}$ \\
\hline $\mathbf{1}$ & 19,97 & 29,08 & 27,46 \\
\hline $\mathbf{2}$ & 23,55 & 28,62 & 28,78 \\
\hline $\mathbf{3}$ & 24,70 & 31,12 & 30,56 \\
\hline
\end{tabular}

Os resultados finais da programação dos diferentes sensores para avaliar o consumo de energia, durante as operações de campo, com o conjunto trator-implemento, apresentaram valores compatíveis com os índices técnicos esperados, mostrando a eficiência do sistema de aquisição de dados proposto. 


\section{Conclusões}

A aquisição de dados em tempo real mostrou-se um recurso bastante eficiente para realizar testes de avaliação de desempenho das operações de campo do conjunto trator-implemento.

O computador de placa única utilizado nesse sistema apresentou alta capacidade de processamento dos sinais oriundos sensores e dos dados gerados, permitindo trabalhar com a quantidade desejada de sensores sem comprometer as medições realizadas. Pode-se ainda utilizar os recursos do computador embarcado para realizar transmissão de dados à distância através de comunicação serial, wireless, ou qualquer outro.

O uso de microcontroladores e computadores embarcados, com auxílio das linguagens de programação $\mathrm{C}$ e $\mathrm{C}++$, permitiu criar aplicações que tornaram possível o registro de importantes grandezas associadas às operações de campo envolvendo máquinas agrícolas, tais como velocidade, patinagem, consumo de combustível e força de tração.

A partir dos resultados obtidos durante os testes em laboratório e dos trabalhos de campo verificou-se que o sistema automático de aquisição de dados, de baixo custo, está apto para auxiliar na tomada de decisões, visando reduzir o uso de energia durante as operações de campo, de um conjunto trator-implemento.

\section{Referências}

GARCIA, R. F.; QUEIROZ, D. M. de; FERNANDES, H. C.; PETERNELLI, L. A. Desempenho operacional de conjunto trator-recolhedora de feijão. Engenharia Agrícola, Jaboticabal, v. 25, n. 1, p. 199-206, jan./abr. 2005.

DOI: 10.1590/S0100-69162005000100022.
GREGO, C. R.; OLIVEIRA, R. P. de; VIEIRA, S. R. Geoestatística aplicada a agricultura de precisão. In: BERNARDI, A. C. de C.; NAIME, J. de M.; RESENDE, A. V. de; BASSOI, L. H.; INAMASU, R. Y. (Ed.). Agricultura de precisão: resultados de um novo olhar. Brasília, DF: Embrapa, 2014. p. 7483.

GRISSO, R.; HELSEL, Z. Optimize wheel slip to save fuel. Extention, 23 mar. 2012. Disponivel em: <http:// articles.extension.org:80/pages/28319/optimize-wheelslip-to-save-fuel>. Acesso em: 5 maio 2017.

HOUMY, K.; CLARKE, L. J.; ASHBURNER, J. E.; KIENZLE, J. Agricultural mechanization in Sub Saharan Africa: guideliness for prreparing a strategy. Rome: FAO, 2013. 93 p. (Integrated Crop Management, 22).

LAMBORELlE, A.; ÁlVAREZ, L. F. Farming 4.0: the future of agriculture? Disponivel em: $<\mathrm{https}: / / \mathrm{www}$. euractiv.com/section/agriculture-food/infographic/ farming-4-0-the-future-of-agriculture/ $>$. Acesso em: 16 jun. 2017.

LILJEDAHL, J. B.; TURNQUIST, P. K.; SMITH, D. W.; HOKI, M. Tractors and their power units. 4. ed. Nova York: Van Nostrand Reinhold, 1989. 463 p.

MANTOVANI, E. C. Compactação do solo. Informe Agropecuário, Belo Horizonte, n. 13, p. 52-55, 1986.

MANTOVANI, E. C.; LEPLATOIS, M.; INAMASSU, R. Y. Automação do processo de avaliação de desempenho de tratores e implementos em campo. Pesquisa Agropecuária Brasileira, Brasília, DF, v. 34, n. 7, p. 1241-1246, jul. 1999.

DOI: 10.1590/S0100-204X1999000700017.

MOITZI, G.; WAGENTRISTL, H.; REFENNER, K.; WEINGARTMANN, H.; PIRINGER， G.; BOXBERGER, J.; GRONAUER, A. Effects of working depth and wheel slip on fuel consumption of selected tillage implements. CIGR Journal, v. 16, n. 1, p. 182190, 2014. 
MOLLOY, D. Exploring BeagleBone: tools and techniques for building with embedded Linux. Indianapolis: John Wiley \& Sons, 2014. 576 p.
RUBHINI, B.; SIBI, S. S. K.; SINDHU, D. D. Role of embedded system in agricultural equipments: a review. IJISET - International Journal of Innovative Science, Engineering \& Technology, v. 1, p. 457-463, 2014. 